\title{
La gestión de la seguridad basada en los comportamientos. ¿Un proceso que funciona?
}

\section{The behavior safety management, a process that works?}

\section{Ciro Martínez Oropesa'}

1. Universidad Autónoma de Occidente, Cali y Universidad Nacional de Colombia. Colombia.

Recibido: 11-09-15

Aceptado: 13-11-15

\section{Correspondencia}

Ciro Martínez Oropesa

Universidad Autónoma de Occidente, Cali. Colombia

Universidad Nacional de Colombia

Correo electrónico: cmartinezo@uao.edu.co; cmartinezo@unal.edu.co

\section{Resumen}

Introducción: El proceso de gestión de la seguridad basada en los comportamientos (PGSBC), se basa en el desarrollo de observaciones a las personas en el cumplimiento de las tareas y retroalimentación de información y reforzamiento positivo en tiempo real, con el propósito de eliminar los comportamientos a riesgos observados, así como, en algunos de los casos más avanzados, modificar los factores ambientales y organizativos que los originan. Esta reflexión se desarrolló con el objetivo de brindar un marco referencial resumido sobre la gestión de la seguridad basada en los comportamientos y aportar datos y fundamentos que permiten resaltar los beneficios e impactos para las empresas. Se realizaron revisiones de una importante información publicada sobre resultados de este proceso. De esta reflexión se deducen y resumen cambios e impactos positivos en la gestión de la seguridad en muchas empresas en las últimas décadas, basada en indicadores proactivos y reactivos de la Seguridad Industrial.

Med Segur Trab (Internet) 2015; 61 (241) 424-435

Palabras clave: Proceso de gestión de la seguridad basada en los comportamientos, Seguridad Industrial, Accidentes Laborales.

\begin{abstract}
The behavior based safety process (PGSBC), is based on observations of people developing tasks and getting feedback in the form of positive reinforcement in real time, with the aim of eliminating risky behaviors observed and, in some of the more advanced cases, to modify the environmental and organizational factors that cause them. This reflection was developed with the aim of providing a summarized framework on behavior based safety and provide data and rationale that highlights the benefits and impacts for businesses. Reviews of important information published on the process results were performed. From this reflection the changes and positive impacts on safety management in many companies in recent decades have been deducted and summarized, based on proactive and reactive indicators of Industrial safety.
\end{abstract}

Med Segur Trab (Internet) 2015; 61 (241) 424-435

Keywords: Behavior based safety process, Safe Behavior, Industrial Safety, Occupational Accidents. 


\section{INTRODUCCIÓN}

En las últimas décadas las organizaciones han venido adoptando diversos modelos de gestión de la seguridad y la salud en el trabajo, con la finalidad de mejorar su asertividad en la disminución de los accidentes e incidentes laborales. Sin embargo, la mayoría de estos modelos de gestión tradicionales han estado limitados por su carácter reactivo y temporal. Uno de los enfoques de mayor importancia en la superación de todas estas limitaciones tiene que ver con en el proceso de gestión de seguridad basada en los comportamientos (PGSBC), que se basa en el desarrollo de observaciones a las personas en el cumplimiento de las tareas y retroalimentación de información y reforzamiento positivo en tiempo real, con el propósito de eliminar los comportamientos y riesgos observados, así como, en algunos de los casos más avanzados, modificar los factores ambientales y organizativos que los originan.

La esencia de este proceso radica en la realización de observaciones enfocadas en situaciones o sobre la base de un inventario de comportamientos críticos utilizados en el desarrollo de las tareas por los empleados. La observación se realiza por un personal que se gestiona y capacita minuciosamente, y luego se responsabiliza con las observaciones sistemáticas de los comportamientos durante la ejecución de las tareas críticas, registrando las acciones seguras y de riesgo que describen el nivel de ejecución. El proceso de gestión de seguridad basada en el comportamiento es, ante todo, un proceso fundamentado en influenciar al comportamiento humano, por medio de un compromiso integral que impulsa la participación de la gerencia, mandos medios, supervisores y empleados en general. La aplicación de este proceso no se limita a un tipo de sector o grupo industrial determinado. Es universal, como otros modelos de gestión, y basa su efectividad no exactamente en la disminución de los accidentes, sino en el aumento de los comportamientos seguros, como la base fundamental para disminuir los accidentes del trabajo.

Es así como este proceso impacta positivamente las tasas de incidentes de la forma más rentable, fundamentado en componentes tales como: a) identificación de los comportamientos inseguros, b) una adecuada observación por medio de observaciones enfocadas en las situaciones de trabajo o a través de listas de verificación, retroalimentando a las personas observadas, y reforzando los comportamientos de aquellos que posee una ejecución segura, c) educando a los observadores y empleados, d) evaluando el comportamiento de seguridad, y e) propiciando una amplia e ilimitada participación en el proceso de cambio cultural y de gestión de la seguridad. ${ }^{1}$

La excelencia de la seguridad requiere de un cambio de cultura importante, que permita una participación activa de los empleados de una compañía, así como un alto compromiso de la alta gerencia en los procesos de transformación, que animan todo tipo de proceso que necesite de estas características.

Existe una amplia pero dispersa información sobre algunas de las experiencias obtenidas con la aplicación de este proceso, y no precisamente en idioma español, por lo que es importante que los interesados en profundizar en estos temas, posean un material de referencia como este que les relacione las mejores experiencias alcanzadas y motive a generalizar su aplicación en cualquier empresa que posea las condiciones más apropiadas.

\section{EL PROCESO DE GESTIÓN DE LA SEGURIDAD BASADA EN LOS COMPORTAMIENTOS (PGSBC)}

La ingeniería tradicional y los enfoques de gestión han enfocado su atención sobre los controles que exige la automatización, el cumplimiento de los procedimientos y de los controles administrativos, y hay que reconocer que aunque en algún momento lograron reducir de manera significativa el número de accidentes, las tasas de incidentes se han mantenido en niveles inaceptables. Las sociedades demandan niveles de vida más elevados y lógicamente esto pasa primero, por no accidentarse, ni enfermarse en el 
trabajo. La fusión de diferentes disciplinas o ciencias no es un concepto nuevo. En 1876, como parte de una cátedra universitaria en filosofía se comenzó a estudiar procesos de comportamientos y del desarrollo de la ciencia de la psicología. Entre las décadas de 1970 y 1980, la fusión de las ciencias del comportamiento en concordancia con la seguridad, determinó el nacimiento de la seguridad basada en el comportamiento. ${ }^{2,3}$

Los primeros intentos de influir en los comportamientos ya se analizaban desde las obras de Herbert William Heinrich. En la década de los años treinta, Heinrich examinó miles de informes de accidentes elaborados por los supervisores, arribando a la conclusión que el $\mathbf{8 8 \%}$ de los incidentes en el lugar de trabajo, eran directamente atribuibles a las acciones inseguras de los trabajadores. Estudios posteriores de DuPont (1956) confirmaron los estudios y hallazgos de Heinrich. Debe señalarse que estos estudios tienen, críticos tenaces. ${ }^{4}$ Entre otros puntos a señalar, los datos de Heinrich no permitieron conocer, las razones que determinaron que las personas actuaran de una forma determinada para causar el accidente, o cómo había ocurrido el accidente. El análisis del comportamiento organizacional se ha hecho durante 100 años, sin embargo, direccionar la investigación aplicada de forma específica hacia la seguridad, ha estado sucediendo hace sólo unas décadas. La frase "Seguridad basada en el comportamiento" fue acuñada por Geller, ${ }^{5-8}$ para luego convertirse en eslogan de los sistemas de seguridad. Aun así, existe una cierta discusión en cuanto al origen del término, algunos dicen que proviene de Geller, pero muchos otros dicen que fue Dan Petersen. La evidencia parece estar a favor de Petersen, quien escribió 17 libros de seguridad antes de su muerte en 2007, y fue probablemente el profesional en seguridad más conocido en los Estados Unidos. En 1978 escribió: «La gestión de la seguridad: Un enfoque humano", en el que Petersen, ${ }^{9-11}$ se refiere a la investigación y la escritura de B. F. Skinner, el padre del análisis de los comportamientos. Es evidente que los análisis de los comportamientos constituyen las bases científicas del campo. Como Krause de forma acertada afirma, "... La frase seguridad basada en el comportamiento se refiere de manera estricta a la aplicación de métodos de análisis del comportamiento para lograr una mejora continua en el funcionamiento de la seguridad». ${ }^{12}$ Hoy existe un amplio número de revisiones que son resultados de amplias investigaciones y han ayudado a enriquecer su abordaje y eficacia.

Se cuenta además con un amplio número de revisiones que son resultados de amplias investigaciones $\mathrm{y}$ han ayudado a enriquecer su abordaje y eficacia. Entre la década del 2000 al 2010, se destacan las realizadas por diversos autores: Sulzer-Azaroff \& Austin, 2000 ${ }^{13}$; Williams \& Geller, 2000 ${ }^{14}$; Sulzer-Azaroff. ${ }^{15}$ Más reciente se conoce de investigaciones que intentan mostrar la relación entre los distintos niveles de "Cultura de la seguridad" de una organización y su efecto sobre el comportamiento seguro. ${ }^{16,17}$ (Glendon \& Litherland, 2001; Dejoy, 2005).

La Dra. Beth Sulzer-Azaroff, una pionera en el campo de la seguridad basada en el comportamiento, publicó en el año 1978 el primero de muchos artículos sobre la seguridad basada en el comportamiento en el diario de Gestión del Comportamiento Organizacional. El artículo fue titulado, «Ecología del comportamiento y la prevención de accidentes». Su capítulo, "Comportamiento enfoques para profesionales salud y seguridad", Sulzer-Azaroff y Frederiksen (1982) en el "Manual Gestión de Comportamiento Organizacional» por Fredericksen, sigue siendo aún en nuestros días una de las mejores explicaciones de la seguridad basada en el comportamiento. ${ }^{17}$ Pero junto a ello hubo una oleada de trabajos a partir de la década de los setenta. Por ejemplo, Fox (1987) en las actividades de la minería a cielo abierto en Utah a partir de 1972, demostraron que con el uso de estrategias de economía (control de pérdidas), se había generado una mejora de los resultados de la seguridad, los cuales se mantuvieron durante más de 12 años. ${ }^{18}$ El proceso de gestión de la seguridad basada en los comportamientos se ha utilizado tradicionalmente en entornos industriales. Sin embargo, como expresa Al-Hemoud (2006): «Una nueva generación ha obtenido éxito con la aplicación del proceso de seguridad basado en los comportamientos, en oficinas y laboratorios...” ${ }^{19}$

La metodología de la seguridad basada en el comportamiento ha sido amplia, experimentada y contrastada desde los años 1980 hasta nuestros días. Sin embargo los 
primeros trabajos y experimentos que utilizan técnicas de modificación de comportamientos surgen desde antes de esta fecha, Bird y Schlesinger (1970). ${ }^{20}$ Existen algunos manuales en varias lenguas que explican sus principios y su metodología práctica (Geller, 2001; Krause, 1990; ${ }^{21}$ López Mena, 1989; ${ }^{22}$ Petersen, 1980 $;{ }^{10}$ Sulzer-Azaroff, 1978,15). Muchos han contribuido en gran medida a la evolución de la práctica en este campo, como por ejemplo McSween (1995), un analista del comportamiento, el autor de valores de seguridad basados en procesos. Mejorando su cultura de la seguridad con un enfoque de comportamientos, McSween (1995) ${ }^{23}$ y Geller, que escribió «Trabajando con Seguridad» en 1996, han tenido un impacto significativo en las últimas dos décadas. Según los aportes hechos por Bandura (1986), este enfoque se ha llevado aún más lejos, desarrollando lo que él denomina la teoría socio-cognitiva. Su teoría destaca en el determinismo recíproco, en el que los determinantes externos de los comportamientos (como las recompensas y castigos) y los determinantes internos (como las creencias, pensamientos y expectativas) forman parte de un sistema de influencias que interactúan afectando a los comportamientos y a otras partes del sistema. ${ }^{24} \mathrm{~A}$ principios de los años 1980 muchos profesionales de seguridad estuvieron motivados en usar las nuevas posibilidades que brindan las ciencias conductuales para mejorar la seguridad industrial en las compañías. Entre las tecnologías investigadas se encontraba la observación conductual. Para Sannino (2007) existen algunos de los conocimientos alcanzados por la psicología de la conducta que resultan aplicables a la seguridad: El primero de ellos es que el comportamiento humano es un fenómeno natural, de ocurrencia frecuente, observable y medible, lo que la convierte en objeto de estudio científico. Además se encuentra directamente relacionado, de forma metódica y predecible con los acontecimientos del medio ambiente. El estudio de la relación entre los comportamientos de las personas y su ambiente, basado en la observación y en un registro sistemático de datos de los comportamientos, constituye el centro de interés de la Psicología de los comportamientos, porque este estudio permite la predicción y administración del comportamiento humano. La relación natural del comportamiento con su medio ambiente señala que las personas aprenden el comportamiento seguro, pero también indica que ellos pueden aprenderlo, de allí que resulta necesario para el prevencionista el conocimiento de los procesos naturales de aprendizaje humano, con el fin de emplear este conocimiento para revertir los aprendizajes inseguros. ${ }^{25}$

\section{IMPLEMENTACIÓN DEL PROCESO DE GESTIÓN DE LA SEGURIDAD BASADO EN LOS COMPORTAMIENTOS}

En muchos años las organizaciones han medido la seguridad por sus índices de fracaso (índice de frecuencia y de gravedad), prácticas de dirección que a toda luz consistían en formas de actuación reactivas por medio de las cuales estas organizaciones han podido hacer muy poco para eliminar los riesgos.

Las organizaciones comenzaban a comprender todas las carencias e inconsistencias que caracterizan el reporte y el no reporte de accidentes, programas incluso bien intencionados pueden convertirse en factores significativos que desestimulan el reporte de accidentes por los empleados. Testimonios de empleados accidentados y testigos a menudo son confusos o deforman los hechos para evitar la culpa. Los datos que se relatan son tanto incompletos como inexactos.

El proceso de gestión de la seguridad basado en los comportamientos profundiza en los actos que causan el accidente, en el lugar de trabajo, el medio ambiente, los equipos, los procedimientos y las actitudes (Al-Hemoud, 2006). ${ }^{19}$ El comportamiento es por definición «un acto observable» y por lo tanto mensurable por la observación en cada lugar de trabajo. Si pudiera establecerse una conexión estadísticamente significativa entre ciertos comportamientos y las probabilidades de los accidentes, midiendo y evaluando estos comportamientos a través de la observación, se podrían proporcionar una argumentación más exacta de la seguridad en cada lugar de trabajo. El proceso de gestión de la seguridad basado en los comportamientos está definido por la consecución de unos 
pasos y procedimientos bien documentados que permiten organizar e integrar las acciones asignadas a cada una de las personas o entes que lo garantizan. Estos pasos deben ser repetidos cíclicamente con el fin de predecir los resultados de forma fiable y de establecer los procesos de mejoramiento continuo que cada nivel demande.

El propósito de la gestión de la seguridad basada en los comportamientos es la identificación y evaluación de los comportamientos y condiciones inseguras en el ambiente de trabajo y el uso de la tecnología actual para:

a. Aumentar el repertorio y la frecuencia de presentación de los comportamientos seguros.

b. Cambiar aquellas condiciones que favorecen la ocurrencia de comportamientos inseguros, aportando con ellos a la reducción significativa de la frecuencia y gravedad de los accidentes en la compañía.

En algunos estudios se ha comprobado que los gerentes y supervisores que apoyan las actividades de seguridad, logran efectos tanto directos como indirectos sobre la cultura de la organización (Zohar, 2002) ${ }^{26}$ De igual forma se ha demostrado que el estilo de liderazgo posee un impacto significativo con relación a la participación en la seguridad, y los líderes pueden fomentar la participación en la seguridad mediante una combinación de influencias tácticas (Clarke \& Ward, 2006). ${ }^{27}$ En contraste con el cambio de los comportamientos, cambiar la cultura con enfoque hacia la seguridad, es más un proceso vertical que se desarrolla desde el nivel gerencial a la base operativa de la compañía. Por consiguiente, la atención se centra en la comprensión cabal del cambio sustentado en nuevos principios, valores y creencias fundamentales de la organización, y trabajando además en importantes modificaciones de las políticas y programas en general, así como la concurrencia de las actividades de gestión que permitan atender las nuevas prioridades, financiamiento e iniciativas, y cambiar las formas de hacer las cosas dentro de la organización. El liderazgo en seguridad es considerado como el proceso de interacción entre los líderes y seguidores, a través del cual los líderes pueden ejercer su influencia en los seguidores para gestionar el cumplimiento de metas de seguridad, bajo las circunstancias de la organización y los factores individuales (Wu, 2005). ${ }^{28}$ El liderazgo es capaz de afectar la actitud hacia la seguridad y la cultura de seguridad de los miembros de su equipo (Flin \& Yule, 2004). ${ }^{29}$ En algunas publicaciones se afirma que el liderazgo y el clima de seguridad son dos importantes factores para predecir un buen rendimiento de seguridad y que este último cumple un papel mediador en la relación entre liderazgo y el logro de seguridad (Wu, 2007). ${ }^{30}$

La gerencia, mandos medios y supervisores han sido reconocidos como los gestores esenciales de la cultura de la seguridad. Dentro de los estilos de liderazgos existentes, el estilo de liderazgo participativo ha sido una de las mejores prácticas para el desarrollo de la cultura de seguridad y las políticas de seguridad en las organizaciones. El estilo de liderazgo participativo que fomenta entre los trabajadores la confianza, el respeto y el compromiso, ha permitido a los trabajadores aceptar la responsabilidad y el compromiso con la seguridad. ${ }^{31}$ Por último Cohen \& Cleveland, realizando trabajos dentro de la industria pesada en América del Norte con diferentes índices de accidentes, llegaron a la conclusión de que los empleados trabajan con más seguridad cuando están involucrados en la toma de decisiones, cuando tienen responsabilidades concretas y razonables, autoridad y metas, y cuando tienen retroalimentación inmediata sobre su trabajo. ${ }^{32}$ De hecho, la gestión descentralizada en todos los niveles no sólo es el mejor predictor de la propensión de los grupos de trabajo a las iniciativas de seguridad, sino que además es el factor más importante en relación con los otros dos factores predictivos de la motivación de los trabajadores hacia la seguridad: la cohesión de equipo de trabajo y la cooperación. La implementación del proceso de gestión de la seguridad basada en los comportamientos, con la importante mediación del liderazgo de la Gerencia, mandos de dirección intermedio y supervisores, reporta importantes beneficios al mejorar el funcionamiento organizacional en general, la conciencia de los empleados, la cultura, las comunicaciones, la participación, la calidad y la productividad. ${ }^{33}$ 


\section{MODELOS Y TÉCNICAS DE ANÁLISIS PARA MODIFICAR LOS COMPORTAMIENTOS A PARTIR DEL ANÁLISIS DE TAREAS}

Existen numerosos modelos que fueron surgiendo a partir de las diferentes investigaciones en el área de la psicología conductual aplicada a la seguridad industrial. Entre los modelos de mayor reconocimiento se encuentra, el modelo Dupont, con el programa Safety Training Observation Program (STOP) cuyo objetivo es prevenir las lesiones laborales mediante habilidades desarrolladas para reconocer y eliminar actos y condiciones inseguras. El modelo de Scott Geller se fundamenta en tres conceptos: la triada segura, el cuidado activo y el enfoque positivo y proactivo de la seguridad. Este modelo es puesto en práctica a través de los siguientes pasos: definir, observar, intervenir y testear (proceso DO IT). El otro modelo importante es el de Terry MsSween, el cual pone la atención sobre la cultura en seguridad para el logro de un proceso de seguridad. ${ }^{29}$ Un modelo interesante de modificación del comportamiento es el que se relaciona con el comportamiento organizacional (Luthans \& Stajkovic, 1999), donde se comienza con la identificación de los comportamientos que requieren cambios, acción que es seguida por la medición, se analizan los antecedentes de comportamiento y las consecuencias contingentes en el contexto relacionado con el comportamiento (consecuencias funcionales) para pasar a las estrategias de intervención apropiada, y finalmente medir y evaluar con el objetivo de comprobar si se obtuvieron los resultados deseados. Si no se han obtenido los resultados, se escoge una nueva estrategia o se repite el proceso. ${ }^{34}$

El análisis funcional recoge información acerca de los antecedentes (A), de los comportamientos (B) y de las consecuencias (C), siglas tomadas del significado de las mismas en idioma Inglés: Antecedents-Behaviour-Consequences. Esta es una técnica de análisis que tiene su origen en la teoría conductista de que todo comportamiento está precedido por un(os) antecedente(s) (también denominados "Disparadores" porque dan la señal para ejecutar el comportamiento) y seguido por una(s) consecuencia(s). Según (Hellriegel, 2009) un antecedente es algo que ocurre antes de un comportamiento y lo estimula, mientras que una consecuencia es el resultado de un comportamiento y puede ser positiva o negativa en término de la consecución de la meta o la tarea. ${ }^{35}$ Según Peterson (1968), el análisis funcional del comportamiento (functional behavior analysis) se cumple por medio del siguiente proceso: a. Realizar una observación sistemática de los comportamientos, como un problema para obtener una línea base de frecuencia de comportamientos. b. Realizar una observación sistemática de las condiciones estimulares antecedentes o consecuentes del comportamiento, con atención especial a los estímulos discriminativos y a los refuerzos. c. Manipulación experimental de la condición que aparece como causal relacionada con el comportamiento problema. d. Realizar observaciones y registros de los cambios que se producen en los comportamientos. ${ }^{11}$

En el registro ABC, según Verdugo (1995), se presentan ciertos inconvenientes: el comportamiento observado requiere un principio y un final claros de forma que pueda aislarse en unidades independientes. Puede resultar complicado cuando se observan comportamientos múltiples (a menos que se dispongan de dispositivos mecánicos adicionales). Resulta difícil calcular el índice de fiabilidad, a menos que las respuestas observadas estén dadas en tiempo real (lo que se consigue dividiendo la sesión en breves intervalos de registros). Y, por último, se requiere la atención completa del observador durante todo el tiempo de sesión de observación. ${ }^{36}$

\section{EL REGISTRO Y ANÁLISIS DE TAREAS}

El registro y análisis de tareas es una variante del registro de eventos, aplicable de forma exclusiva en el caso de los procesos de adquisición y análisis de los comportamientos nuevos, desarrollados mediante la técnica de análisis de tareas, consistente en evaluar la ejecución del sujeto en los pasos que conforman la ejecución de una tarea específica. Para llevarlo a cabo, se escribe en la ficha de registro el listado de pasos a ejecutar con la 
valoración que el observador hace de la ejecución del sujeto, confrontándola con el criterio de éxito predeterminado que puede incluir aspectos tales como calidad de ejecución, tiempo empleado y la propia seguridad entre otros. Dicha valoración puede ser dicotómica (paso realizado/ no realizado) o representar un continuum que el observador califica según la ejecución se acerque al criterio (realizado correctamente, con seguridad, etc.).

\section{LAS OBSERVACIONES CONDUCTUALES}

Las observaciones conductuales pretenden ayudar a solucionar el problema, añadiendo una métrica nueva que se sustenta la observación por muestreo, con el objeto de complementar indicadores de análisis retrospectivo de accidentes. El problema con esta nueva tecnología consiste en su carácter subjetivo, ya que se pide a los observadores emitir un juicio de valor sobre la seguridad relativa en los comportamientos que ellos observan. Algunos sistemas solicitan al observador "cuantificar» la seguridad en una escala de 1-10, mientras los otros exigen calificarlos como seguros o inseguros; tales evaluaciones varían deliberadamente entre observadores, lo que limita el éxito de las mismas.

La necesidad de controlar la subjetividad de las observaciones conductuales, demanda que se establezcan pautas conductuales de importancia para enfocar el trabajo de los observadores, y una adecuación previa de las listas de comprobación. En efecto al principio se requerían observaciones más complejas, que demandaban de un mayor conocimiento por parte del observador, mayor entrenamiento y tiempo para los procesos de observación, dependiendo del número de tareas que estaban siendo observadas. En el proceso de transformación se requerirá en primera instancia plantearse la pregunta de si en realidad, las tareas que estaban siendo observadas eran las más importantes. Este aspecto es superado a través de la aplicación del análisis de Pareto, en una hoja de trabajo con los comportamientos más comunes y operacionalmente definidos, por medio de los cuales una organización puede analizar sus accidentes y datos acerca de los comportamientos que tienen la mayor importancia en la reducción potencial de los accidentes basada en datos históricos.

Por otra parte, la utilización de una lista de comprobación para observaciones basadas en los pocos significativos obtenidos por medio del análisis de Pareto, aunque bien permite a los observadores definir los comportamientos estadísticamente más significativos, no deja de ser un análisis ordinario y que a menudo no incluye comportamientos importantes que se incluyan en el análisis de datos sobre los accidentes.

Las listas de comprobación de carácter específicas raras veces incluían las tareas o comportamientos específicos, conectados a la maquinaria o procesos específicos de una industria. Las observaciones contenidas en estas listas de comprobación eran demasiado prolongadas y requerían de un entrenamiento muy extenso, en cursos especiales para observadores.

El tiempo de duración de la observación depende en mayor grado, de la cantidad de comportamientos críticos contenidos en las listas de comprobación, y además del nivel de entrenamiento de los observadores. Sin embargo, con el objetivo de que los comportamientos excluidos no comiencen a condicionar problemas recurrentes, será importante mantener observaciones basadas en las situaciones que rodean cada una de las actividades observadas. ${ }^{37}$

\section{TÉCNICAS DE MODIFICACIÓN DEL COMPORTAMIENTO}

Entre las técnicas de intervención psicológica, basadas en la psicología conductual, dirigidas a modificar o cambiar determinados comportamientos laborales, previamente definidos, se encuentra el refuerzo positivo. Si bien el procedimiento de reforzamiento de los comportamientos es conocido desde hace algunas décadas como parte del repertorio 
de intervención del análisis y modificación de los comportamientos, las experiencias de su aplicación en la industria no son muy amplias.

Cuando los comportamientos van seguidos por las consecuencias que el individuo considera deseables, es más probable que aquellos comportamientos se repitan en el futuro. A este comportamiento se denomina reforzamiento. Cuando las consecuencias son negativas, es poco probable que se repita el comportamiento.

En este contexto pudieran resumirse algunas de las relaciones más significativas que expresan esta interconexión entre los dos procesos: Usar consecuencias que aumentan la frecuencia de los comportamientos. Estas consecuencias se conocen como reforzadores o recompensas. Usar consecuencias que disminuyen la frecuencia de los comportamientos. Estas consecuencias se conocen como castigos. No usar ninguna consecuencia, y así disminuir unos comportamientos aprendidos con anterioridad. La ausencia de consecuencias se usa en el proceso de extinción de los comportamientos.

Para Hellriegel (2009) una contingencia del reforzamiento es la relación que existe entre un comportamiento y los hechos anteriores y posteriores que ocurren en el entorno e influyen en ese comportamiento, implica que se presentará una experiencia agradable después que ha ocurrido un comportamiento deseado y la recompensa es algo que una persona valora como deseable o agradable. ${ }^{35}$

En la psicología preventiva, se han agrupado los recursos que provee el análisis de los comportamientos en diferentes métodos para la motivación hacia la seguridad, para que estos métodos funcionen de forma correcta requieren de datos de los comportamientos observables y medibles que permitan establecer el índice, tasa o indicador en que los empleados se acogen a los métodos o formas más seguras de realizar el trabajo. Ejemplo: el promedio de veces que los empleados usan los medios de protección colectiva en su equipo, pudiendo generar información comparable entre un periodo y otro, antes y después de la intervención respectiva.

\section{EFECTIVIDAD DEL PROCESO DE GESTIÓN DE LA SEGURIDAD BASADO EN LOS COMPORTAMIENTOS}

El proceso de gestión de la seguridad basado en los comportamientos ha demostrado su efectividad a lo largo de su existencia. Se reporta incluso en algunos textos que se puede alcanzar más de un 75\% de reducción del número de accidentes (Austin 1996; ${ }^{38}$ Geller, 2002;7 Krause, 1997; ${ }^{12}$ Laitienen \& Ruohomaki, 1996; ${ }^{39}$ Montero, 1995; ${ }^{40}$ Montero, 2003; ${ }^{41}$ Ray \& Bishop, 1997). ${ }^{42}$

Sin lugar a dudas que, cuando estos comportamientos son bien influenciados, se logran cambios importantes en los resultados que aportan los indicadores de la accidentalidad e incidentalidad, mejoramiento de la moral, la comunicación y el sentido de coherencia dentro de la organización (Peters \& Waterman, 1982; ${ }^{43}$ Senge, 1990). ${ }^{44}$

A principios de los años 1980 muchos profesionales de seguridad estuvieron motivados en usar las nuevas posibilidades que brindaban las ciencias conductuales para mejorar la seguridad industrial en las empresas. Una de las primeras experiencias fue revelada de un estudio que reportaba una reducción en la tasa de frecuencia de 53.8 a 10 lesiones por millón de horas-hombres trabajadas. ${ }^{1}$

Según Sulzer-Azaroff \& Austin, ${ }^{15}$ al investigar en 33 estudios de casos publicados, encontraron que en 32 de ellos disminuyeron las lesiones, en un rango entre 2 y $85 \%$. Por otra parte, el seguimiento de 73 compañías donde se aplicó esta tecnología, demostró una reducción significativa y progresiva de la tasa de lesiones en un período de 5 años. Aunque estos resultados no pueden demostrarse claramente como un efecto directo del uso de esta tecnología, hay bastantes razones para suponerlo (Krause, 1999). ${ }^{45}$ 
El seguimiento de tres de los sitios donde se aplicó esta tecnología, demostró el logro de una reducción significativa de la tasa de lesiones en un período de 1 año. Los accidentes se redujeron a cero. No se tomó ninguna otra medida que influyera en este resultado. ${ }^{40}$

De acuerdo a un análisis por países, se revisaron 24 estudios elaborados en EEUU, encontrando que en todos los casos se reducían los accidentes (Chhokar \& Wallis, 1984). ${ }^{46}$ Un estudio en el Reino Unido reportó una disminución del $21 \%$ en la tasa de accidentes y del $74 \%$ en aquellos directamente asociados a los comportamientos, así como un ahorro de más de 180000 libras esterlinas en una fábrica de teléfonos celulares (Fleming \& Lardner, 2002). ${ }^{47}$ En sitios industriales de Cuba y Colombia, en los cuales se ha introducido esta tecnología, se han logrado disminuciones entre el 60 y el $95 \%$ del número de accidentes por año, tomando como base un período de comparación de 2 años a partir del momento en que es implementada.

De acuerdo a la experiencia específica, obtenida por medio de la investigación desarrollada en empresas de manufactura se obtuvieron importantes decrecimientos de los índices de accidentalidad, después de implementar el nuevo modelo de gestión de la seguridad, con la participación de supervisores en un grupo experimental y varios grupos de control que implementaron el proceso de gestión de la seguridad basado en comportamientos, en diferentes empresas. ${ }^{48}$ Ver tabla 1.

Tabla 1. Comparación de resultados (tasa de accidentes) por estudios publicados

Resultados de diversos estudios (Austin $e t$ Disminución de accidentes 25,0\%.

al., 1996; Geller, 2002; Krause et al., 1997;

Laitienen \& Ruohomaki, 1996; Montero, 1995;

Montero, 2003; Ray \& Bishop, 1997).

Una de las primeras experiencias. (Komaki $e t$ al., 1978).

En 33 estudios de casos publicados (Sulzer-

Azaroff \& Austin, 2000).

El seguimiento de 73 compañías donde se aplicó

esta tecnología (Krause et al., 1999).

El seguimiento de 3 compañías donde se aplicó

accidentes en un año.

esta tecnología (Montero, 1995).

En todos los casos se reducían los accidentes.

Se revisaron 24 estudios desarrollados en EE.UU.

(Chhokar \& Wallis, 1984).

Un estudio en el Reino Unido (Fleming \&

Lardner, 2002).
Una disminución de: 53,8 a 10,2 y un $85 \%$

(accidentes/1.000.000 horas trabajadas).

Significativa y progresiva en 5 años de seguimiento.

En sitios industriales de Cuba y Colombia en los cuales se ha introducido esta tecnología.

Una disminución del $21 \%$ en la tasa de accidentes y del $74 \%$ en aquellos directamente asociados a los comportamientos.

Se han logrado disminuciones entre el $60 \mathrm{y}$ el $95 \%$ del número de accidentes por año.

Empresas que integraron los grupos de control (Martínez; 2014).

Una disminución del 44,4\%, de accidentes con y sin lesiones.

Disminución de 79 a 48 (accidentes/1.000 horas trabajadas)

Número de accidentes por trabajador: 0,1779.

Grupo Experimental (primer semestre)

(Martínez; 2014)

\section{5 accidentes.}

57,6 (accidentes/1.000 horas trabajadas).

Número de accidentes por trabajador: 0,1296.

Grupo Experimental (segundo semestre)

(Martínez; 2014)

\section{2 accidentes.}

33 (accidentes/1.000 horas trabajadas).

Número de Accidentes por trabajador: 0,037. 


\section{CONCLUSIONES}

Existe una tendencia relativamente nueva pero creciente de conocimientos sobre la concienciación de los efectos de las técnicas y procesos de gestión de la seguridad basados en el comportamiento. A pesar de que sus principios no son recientes, la aplicación organizada a la seguridad y la salud es particularmente contemporánea.

Es de interés del autor, mejorar la comprensión básica de la seguridad basada en los comportamientos, así como proporcionar referencias que podrían ser utilizadas para proponer a las compañías interesadas, maximizar los beneficios del proceso de gestión de la seguridad industrial y contrarrestar las pocas limitaciones que puede haber presentado el modelo durante todo estos años.

Se ponen de relieve los beneficios que alcanzan las compañías después de implementar el nuevo modelo de gestión de la seguridad, mostrando importantes decrecimientos de los índices de accidentalidad. Uno de estos beneficios, es la reafirmación de los resultados positivos del modelo de gestión de la seguridad basado en los comportamientos, ya que en las distintas fases del proceso de investigación y en las compañías se evidenciaron crecimientos importantes del nivel de éxito de seguridad durante la investigación, quedando demostrada la generalidad y funcionalidad de las técnicas, así como el modelo argumentado para mejorar la seguridad en cada lugar de trabajo.

Otro aspecto importante desde una perspectiva macro de la gestión, es que existen dos formas que podrán afectar el nivel de éxito en seguridad: una de ellas transita desde la cultura de seguridad, mientras que la otra parte del estado del liderazgo de seguridad, hasta el éxito de la seguridad. Para transformar la cultura en seguridad, será necesario que en el mismo sentido se modifiquen los comportamientos de líderes y trabajadores encargados de gestionar el proceso de seguridad; un proceso acompañará al otro, consolidándose el uno al otro.

El intercambio de información sobre seguridad, entre el supervisor y los empleados, tiene una relación significativa con el éxito en seguridad y el desarrollo de una labor proactiva en prevención, con mejores resultados que en cualquier otra oportunidad. Así mismo, había favorecido la comunicación entre los empleados al aumentarse los reforzamientos positivos para modificar los comportamientos peligrosos.

Desde una perspectiva teórica, estos resultados permiten extender nuestra actual comprensión de las técnicas de modificación de los comportamientos en el contexto de trabajo. Los mecanismos psicológicos que facilitan los cambios en el comportamiento individual dentro de entornos de trabajo aún están sin explorar suficientemente en la literatura. Por lo que en ese contexto, se considera que los resultados de este trabajo amplían la comprensión actual de las técnicas y condiciones que facilitan los cambios de comportamientos humanos en determinados ambientes laborales.

Los resultados también ofrecen una vía para generar futuras investigaciones que examinen otros procesos psicológicos que operan en el marco de la interacción antecedentes, comportamientos y consecuencias.

\section{REFERENCIAS BIBLIOGRÁFICAS}

1. Martínez Oropesa, Ciro. (2014). El Proceso de Gestión de la Seguridad Basada en los Comportamientos: Actuación de los Supervisores en Empresas de Manufactura. Tesis Doctoral. Universidad de León. España.

2. Komaki, J., Barwick, K. D. \& Scott, L. R. (1978). A behavioral approach to occupational safety: Pinpointing and reinforcing safe performance in a food manufacturing plant. Journal of Applied Psychology, 63, 424-445.

3. Krause, T.R., Hidley, J.H., Lareau, W., 1984. Behavioral science applied to industrial accident prevention

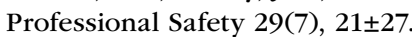

4. Manuele, F.A. (2005) On the Practice of Safety, (3a ed.), USA. John Wiley \& Sons, Inc., Hoboken, NJ, 469 p. 
5. Geller, E.S. (1991). If only more would actively care. Journal of Applied Behavior Analysis, 24, 607-612.

6. Geller, E. S. (2001). Working safe: How to help people actively care for health and safety. (2a ed.). New York: Lewis.

7. Geller, E. (2002). The participation factor: How to increase involvement in occupational safety (1a ed.) pp. 53-97. Illinois, USA: American Society of Safety Engineers.

8. Geller, E. (2005). Behavior-based safety and occupational risk management. Behavior Modification, 29(3), 53-56.

9. Petersen, D. (1996). Safety by objectives: What gets measured gets done. New York, United States: Van Nostrand Reinhold (2a ed.) 217-226

10. Petersen, D. (1980). Analyzing Safety Performance. New York: Garland Publishing.

11. Peterson, D. R. (1968). The clinical study of social behavior. New York: Appleton-Century-Crofts.

12. Krause, T., Hidley, J. \& Hodson, S. (1997). The behavior-based safety process: Managing involvement for an injury free culture (2a ed.) pp. 127-142. New York: Van Nostrand Reinhold.

13. Sulzer, A. \& Austin J. (2000). Does BBS work? Behavior-based safety and injury reduction: A survey of the evidence. Professional Safety, 45(7), 19-24.

14. Williams, J. \& Geller, E. (2000). Behavior based intervention for occupational safety: Critical impact of social comparison feedback. Journal of Safety Research, 31(3), 135-142.

15. Sulzer-Azaroff, B. (1978). Behavioral ecology and accident prevention. Journal of Organizational Behavior Management, 2, 11.

16. Glendon, A. I. \& Litherland, D. K. (2001). Safety climate factors, group differences, and safety behavior in road construction. Safety Science, 39, 157-188.

17. DeJoy, D.M. (2005). Behavior change versus culture change: Divergent approaches to managing workplace safety. Safety Science, 43, 105-129.

18. Sulzer, A. (1982). Behavioral Approaches to Occupational Health and Safety. 515-531. In Frederiksen, L. W. (Ed.), Handbook of Organizational Behavior Management. New York: Wiley.

19. Al-Hemoud, A. M. \& Al-Asfoor, M. M. (2006). A behavior based safety approach at a Kuwait research institution. Journal of Safety Research, 37 (2), 2001-2006.

20. Bird, F.E., \& Schlesinger, L.E. (1970). Safe-behavior reinforcement. American Society of Safety Engineer Journal, 15, 16-24.

21. Krause, T.R., Hidley, J.H. \& Hodson, S.J. (1990). The Behavior Based Safety Process. Managing Involvement for an Injury-Free Culture. New York: Van Nostrand Reinhold.

22. López Mena, L. (1989). Intervención psicológica en la empresa. Barcelona. Martínez Roca.

23. McSween, T.E. (1995). The Values-Based Safety Process: Improving Your Safety Culture with a BehavioraL Approach. New York: Van Nostrand Reinhold.

24. Bandura A. (1986). Social Foundations of thought and action: A social cognitive theory, Englewood Cliffs, NJ: Prentice Hall.

25. Sannino, B.D. (2007). Motivación para la seguridad del trabajo, basada en la conducta. VII Taller de Seguridad y Salud Ocupacional. Mejorando la Conducta en Seguridad. Expocorma. Concepción. Chile.

26. Zohar, D. (2002). Modifying supervisory practices to improve sub-unit safety: A leadership-based intervention model. Journal of Applied Psychology, 87, 156-163.

27. Clarke, S. \& Ward, K. (2006). "The Role of Leader Influence Tactics and Safety Climate in Engaging Employees' Safety Participation," Risk Analysis, 26(5), 1175-1185.

28. Wu, T. (2005). The validity and reliability of safety leadership scale in universities of Taiwan. International Journal of Technology and Engineering Education, 2(1), 27-42.

29. Flin, R. \& Yule, S. (2004). «Leadership for Safety: Industrial Experience,» Qual Saf Health Care, 13, 180-184

30. Wu, T., Liu, C. \& Lu, M. (2007). Safety climate in university and college laboratories: Impact of organizational and individual factors. Journal of Safety Research, 38(1), 91-102.

31. O'dea, A. \& Flin R. (2001). "Site managers and safety leadership in the offshore oil and gas industry," Safety Science, 37(1), 39-57.

32. Cohen, H. \& Cleveland, R. (1983). Safety program practices in record-holding plants. Professional Safety, 28, 26-33.

33. Krause, T. R. (2002). Cross-Functional Improvement: Behavior-Based Safety as a Tool for Organizational Success. Professional Safety, Vol. 47. No. 8, 27-3. 
34. Luthans, F. \& Stajkovic, A. (1999). Reinforce for Performance: The Need to Go beyond Pay and Even Rewards. Vol. 13, No. 2, Themes: Technology, Rewards, and Commitment (May, 1999), pp. 49-57.

35. Hellriegel, D. (2009). Comportamiento organizacional. (12a ed.) México: Cengage Learning Editores.

36. Verdugo Alonso, M.A. (1995). Personas con discapacidad: perspectivas psicopedagógicas y rehabilitadoras. Siglo XXI de España Editores.

37. Martinez Oropesa, Ciro. (2014). El Proceso de Gestión de la Seguridad Basada en los Comportamientos: Actuación de los Supervisores en Empresas de Manufactura. Tesis Doctoral. Universidad de León. España.

38. Austin, J., Kessler, M., Riccobono, J. \& Bailey, J. (1996). Using feedback and reinforcement to improve the performance and safety of a roofing crew. Journal of Organizational Behavior Management, 16(2), 49-75.

39. Laitienen, H. \& Ruohomaki, I. (1996). The effects of feedback and goal setting on safety performance at two construction sites. Safety Science, 24, 61-73.

40. Montero, R. (1995). Psicosociología preventiva aplicada a la accidentabilidad laboral. Estudios Empresariales, 88(2), 64-68.

41. Montero, R. (2003). Siete principios de la Seguridad Basada en los Comportamientos. Prevención, Trabajo y Salud, 25, 4-11.

42. Ray, P. \& Bishop, P. (1997). Efficacy of the components of a behavioral safety program. International Journal of Industrial Ergonomics, 19, 19-29.

43. Peters, T. \& Waterman, R. (1982). In Search of Excellence. London: Harper Collins.

44. Senge, P. (1990). The Fifth Discipline: The Art and Practice of the Learning Organization. Sydney: Random House.

45. Krause, T.R. Seymour, K.J. (1999). Long-term evaluation of a behavior-based method for improving safety performance: a meta-analysis of 73 interrupted time-series replications. Safety Science, 32, 1-18.

46. Chhokar, J. S., \& Wallin, J. A. (1984). A field study of the effect of feedback frequency on performance. Journal of Applied Psychology, 69(3), 524-530.

47. Fleming, M. \& Lardner, R. (2002). Strategies to promote safe behaviour as part of a health and safety management system. HSE Contract Research Report CRR430, HSE Books.

48. Martínez Oropesa, C. (2014). El Proceso de Gestión de la Seguridad Basada en los Comportamientos: Actuación de los Supervisores en Empresas de Manufactura. Universidad de León. León y Castilla. España. 